\title{
Activation of synovial fibroblasts from patients at revision of their metal-on-metal total hip arthroplasty
}

Jing $\mathrm{Xu}^{1,2+}$, Junyao Yang ${ }^{2,3+}$, Jian Chen ${ }^{4}$, Xiaoli Zhang ${ }^{2}$, Yuanhao Wu ${ }^{2}$, Alister Hart ${ }^{5}$, Agata Nyga ${ }^{6,7^{*}}$ (D) and Julia C. Shelton ${ }^{2^{*}}$

\begin{abstract}
Background: The toxicity of released metallic particles generated in metal-on-metal (MoM) total hip arthroplasty (THA) using cobalt chromium ( $\mathrm{CoCr}$ ) has raised concerns regarding their safety amongst both surgeons and the public. Soft tissue changes such as pseudotumours and metallosis have been widely observed following the use of these implants, which release metallic by-products due to both wear and corrosion. Although activated fibroblasts, the dominant cell type in soft tissues, have been linked to many diseases, the role of synovial fibroblasts in the adverse reactions caused by CoCr implants remains unknown. To investigate the influence of implants manufactured from $\mathrm{CoCr}$, the periprosthetic synovial tissues and synovial fibroblasts from patients with failed MoM THA, undergoing a revision operation, were analysed and compared with samples from patients undergoing a primary hip replacement, in order to elucidate histological and cellular changes.

Results: Periprosthetic tissue from patients with MoM implants was characterized by marked fibrotic changes, notably an increase in collagen content from less than $20 \%$ to $45-55 \%$, an increase in a-smooth muscle actin positive cells from 4 to $9 \%$ as well as immune cells infiltration. Primary cell culture results demonstrated that MoM synovial fibroblasts have a decreased apoptosis rate from 14 to $6 \%$ compared to control synovial fibroblasts. In addition, synovial fibroblasts from MoM patients retained higher contractility and increased responsiveness to chemotaxis in matrix contraction. Their mechanical properties at a single cell level increased as observed by a $60 \%$ increase in contraction force and higher cell stiffness (3.3 $\mathrm{kPa}$ in MoM vs $2.18 \mathrm{kPa}$ in control), as measured by traction force microscopy and atomic force microscopy. Further, fibroblasts from MoM patients promoted immune cell invasion by secreting monocyte chemoattractant protein 1 (MCP-1, CCL2) and induced monocyte differentiation, which could also be associated with excess accumulation of synovial macrophages.

(Continued on next page)
\end{abstract}

\footnotetext{
* Correspondence: agata.nyga@ucl.ac.uk; j.shelton@qmul.ac.uk

†Jing Xu and Junyao Yang contributed equally to this work.

${ }^{6}$ Research Department of Surgical Biotechnology, Division of Surgery and Interventional Sciences, University College London, London NW3 2QG, UK ${ }^{2}$ Institute of Bioengineering, School of Engineering and Materials Science, Queen Mary University of London, London, UK

Full list of author information is available at the end of the article
}

C C The Author(s). 2020 Open Access This article is licensed under a Creative Commons Attribution 4.0 International License, which permits use, sharing, adaptation, distribution and reproduction in any medium or format, as long as you give appropriate credit to the original author(s) and the source, provide a link to the Creative Commons licence, and indicate if changes were made. The images or other third party material in this article are included in the article's Creative Commons licence, unless indicated otherwise in a credit line to the material. If material is not included in the article's Creative Commons licence and your intended use is not permitted by statutory regulation or exceeds the permitted use, you will need to obtain permission directly from the copyright holder. To view a copy of this licence, visit http://creativecommons.org/licenses/by/4.0/ The Creative Commons Public Domain Dedication waiver (http://creativecommons.org/publicdomain/zero/1.0/) applies to the data made available in this article, unless otherwise stated in a credit line to the data. 


\begin{abstract}
(Continued from previous page)
Conclusion: Synovial fibroblasts exposed in vivo to MoM THA implants that release $\mathrm{CoCr}$ wear debris displayed dramatic phenotypic alteration and functional changes. These findings unravelled an unexpected effect of the $\mathrm{CoCr}$ alloy and demonstrated an important role of synovial fibroblasts in the undesired tissue reactions caused by MoM THAs.
\end{abstract}

Keywords: Cobalt chromium debris, Synovial fibroblast, Inflammation, Fibrosis

\section{Background}

Soft tissue reactions develop in response to all total hip replacements, however between 2008 and 2015 in the UK, $92.5 \%$ of hip revision procedures involved metal-on-metal (MoM) total hip arthroplasty (THA) implants [1], manufactured from a cobalt chromium alloy $(\mathrm{CoCr})$. The formation of pseudotumours, aseptic lymphocyte-dominated vasculitis-associated lesion (ALVAL) and metallosis (often defined as aseptic fibrosis and local necrosis) are believed to be the sequelae of large amounts of $\mathrm{CoCr}$ metal particles and dissociated ions released from the hip bearing surfaces due to both wear processes and corrosion [2-4]. Synovial tissues retrieved at the MoM revision surgery have shown the presence of a large number of amorphous particles predominantly composed of chromium and cobalt [5-7]. The majority of these particles from the implants are in the nano-sized scale and the severity of the adverse tissue reactions is reported to be related with the complexity of the particles and blood metal ion levels [7]. The spectrum of these tissue reactions is extensive and ranges from small asymptomatic cysts to destructive periprosthetic soft-tissue masses, which may lead to severe symptoms of pain and ultimately implant failure [8]. The integrity of the synovial lining is often impaired by inflammatory cell infiltrates (lymphocytes, macrophages, plasma cells and giant cells), which indicate the extent of ALVAL [9]. While immune cells play a key role in the initiation and progression of the tissue reactions $[1,10]$, some evidence suggests that chronic inflammation occurs because of the activated fibroblasts, resulting in the inappropriate survival and retention of immune cells within inflamed tissue [11]. However, the role of stromal fibroblasts and tissue/extracellular matrix (ECM) organisation in the inflammatory response following exposure to cobalt metal wear and corrosion products in vivo remains largely unknown.

Previously, we reported that metal ions, particularly cobalt, alter healthy human dermal fibroblasts in vitro by stimulating contraction and the release of pro-fibrotic signals from macrophages, thus leading to fibrotic reactions and matrix remodelling [12]. To further investigate whether this interaction between fibroblasts and immune cells also occurs in vivo following exposure to $\mathrm{CoCr}$ particulates and associated ions, we examined ex vivo the properties of fibroblasts isolated from periprosthetic synovial tissues from patients implanted with a MoM (manufactured from standard $\mathrm{CoCr}$ alloy) THA, retrieved during revision operations. To uncouple the role of any underlying inflammatory condition, we used a control group of synovial fibroblasts isolated from patients undergoing a primary hip replacement operation. As soft tissue reactions have not been reported as often in patients with either ceramic-on-polyethylene or metal-on-polyethylene THA bearings [1], fibroblasts from these patients were not investigated. We hypothesise that the synovial fibroblasts retrieved at revision operations would show altered mechanical and functional properties that could be related to the released wear and corrosion products from the bearing surfaces of the MoM THAs, and could thereby stimulate inflammatory responses in vitro. The implication of this study suggests that synovial fibroblasts have a role in the in vivo inflammatory soft tissue reactions following MoM THA.

\section{Materials and methods Sample collection \\ Ethics approval and patient selection}

Ethics approval [07/Q0401/25, West London Research Ethics Committee] and written patient consent was obtained for the use of tissue samples (synovial membranes) removed during surgery. Tissue samples were collected from patients (Table 1) undergoing either primary hip replacement surgery (Primary THA) or a revision surgery where a primary MoM hip implant had been used (Revision THA). All tissue specimens were anonymised. Patients with unilateral and bilateral total hip replacements were included in this study, whilst patients with prostheses other than MoM, were excluded from this study. The criteria for failed MoM implants included unexplained pain, implant loosening and high cobalt and chromium ion levels in the blood, as described in Table 1. The original reason for surgery for the revision THAs would have been OA. The exclusion criteria in this study were infection, mechanical instability or prosthesis malalignment.

\section{Tissue harvest and storage}

Primary fibroblasts were isolated from biopsies obtained from patients once the joint capsule was exposed during surgery. Another part of the tissues used for histology 
Table 1 Summary of patient demographics

\begin{tabular}{|c|c|c|c|c|c|}
\hline & No. & $\begin{array}{l}\text { Gender } \\
(\mathrm{M} / \mathrm{F})\end{array}$ & $\begin{array}{l}\text { Age } \\
\text { (year) }\end{array}$ & Time from primary to revision (year) & Reason for surgery \\
\hline \multirow[t]{8}{*}{ Primary THA } & 1 & $\mathrm{~F}$ & 53 & I & $\mathrm{OA}$ \\
\hline & 2 & $\mathrm{~F}$ & 53 & / & $\mathrm{OA}$ \\
\hline & 3 & $\mathrm{~F}$ & 54 & / & $\mathrm{OA}$ \\
\hline & 4 & $\mathrm{~F}$ & 56 & / & $\mathrm{OA}$ \\
\hline & 5 & M & 49 & / & $\mathrm{OA}$ \\
\hline & 6 & $\mathrm{~F}$ & 58 & / & $\mathrm{OA}$ \\
\hline & 7 & $\mathrm{~F}$ & 62 & / & $\mathrm{OA}$ \\
\hline & 8 & $\mathrm{~F}$ & 55 & / & $\mathrm{OA}$ \\
\hline \multirow[t]{8}{*}{ Revision THA } & 1 & $\mathrm{~F}$ & 85 & 10 & Painful hip with elevated metal ions \\
\hline & 2 & $\mathrm{~F}$ & 66 & 5 & Painful hip \\
\hline & 3 & M & 63 & 14 & Painful hip \\
\hline & 4 & $\mathrm{~F}$ & 90 & 7 & Trunionosis \\
\hline & 5 & M & 75 & 6 & Painful hip with elevated metal ions \\
\hline & 6 & M & 75 & 11 & Painful hip \\
\hline & 7 & $\mathrm{~F}$ & 56 & 9 & Aseptic loosening \\
\hline & 8 & M & 79 & 9 & Elevated metal ions \\
\hline
\end{tabular}

(OA osteoarthritis)

was stored in 10\% Neutral Buffered Formalin (Sigma, UK) at room temperature before processing.

\section{Histology}

Fixed tissues were dehydrated through a series of graded ethanol baths (70,90\%, absolute ethanol), cleared in a xylene bath (Sigma, UK) and finally embedded in paraffin and placed on ice at $-20^{\circ} \mathrm{C}$ overnight. The tissue in the wax blocks was trimmed and cut into $5 \mu \mathrm{m}$ sections (Accu-Cut ${ }^{\circ}$ SRMTM 200 rotary microtome, Torrance, CA, USA). Slides were deparaffinised by washing in 2 changes of xylene and graded ethanol baths (absolute ethanol, 90, 70\%). Antigen retrieval was performed to unmask the antigenic epitope of the tissue sample by boiling the deparaffinised sections in citrate buffer at $\mathrm{pH}$ 6.0. Endogenous peroxidase activity was blocked by incubating sections in $3 \% \mathrm{H}_{2} \mathrm{O}_{2}$ solution (Sigma, UK) in $\mathrm{PBS}$ at room temperature for $10 \mathrm{~min}$ followed by 2 rinses in PBS. To reduce background staining the samples were incubated with normal goat serum $(5 \%$ in PBS) to block nonspecific binding sites. After removing the blocking buffer, $100 \mu \mathrm{l}$ of rabbit polyclonal anti-typeI collagen antibody (1:1000 in $1 \%$ goat serum in PBS, Abcam, UK) or rabbit polyclonal anti- $\alpha$-smooth muscle actin (SMA) antibody (1:2000; Sigma-Aldrich, USA) was added to the sections on the slides and incubated in a humidified chamber at room temperature for $1 \mathrm{~h}$, after which the slides were washed twice in PBS. $100 \mu$ of a diluted biotinylated secondary antibody (in 1\% goat serum in PBS, Vector Laboratories, UK) was then applied to the sections and incubated in a humidified chamber at room temperature for $30 \mathrm{~min}$, washed in PBS, then incubated for $30 \mathrm{~min}$ with VECTASTAIN ${ }^{\circ}$ ABC Reagent (Vector Laboratories, UK). Samples were washed and incubated in peroxidase substrate solution (Vector Laboratories, UK) with positive staining visualized by using a DAB Peroxidase (HRP) Substrate Kit (Vector laboratories, UK).

\section{Isolation and culture of synovial fibroblasts}

Biopsy samples were collected in DMEM medium, then transported on ice, and primary fibroblasts were isolated within $6 \mathrm{~h}$. The harvested tissues were transferred into a $10 \mathrm{~cm}$ tissue culture dish in DMEM medium using a sterile forceps and finely minced into approximately $1 \mathrm{~mm}^{3}$ pieces using sterile scalpels. The pieces of tissues were placed in a $15 \mathrm{ml}$ centrifuge tube containing $10 \mathrm{ml}$ of digestion solution $(0.1 \%$ collagenase in DMEM medium, Sigma, UK) and incubated at $37^{\circ} \mathrm{C}$ under rotation for $2 \mathrm{~h}$. $15 \mathrm{ml}$ DMEM medium was subsequently added to dilute the collagenase which was passed through a $70 \mu \mathrm{m}$ cell strainer (BD Biosciences, UK) to obtain a single-cell suspension. The cell suspension was centrifuged for $5 \mathrm{~min}$ at $200 \mathrm{x} \mathrm{g}$ at room temperature, the supernatant was discarded and the pellet resuspended in DMEM cell culture medium (10\% FBS and $1 \% \mathrm{P} / \mathrm{S})$. Harvested cells were counted and seeded at approximately 4000 cells $/ \mathrm{cm}^{2}$ in a T75 flask. The human synovial fibroblasts were cultured at $37^{\circ} \mathrm{C}$ and $5 \% \mathrm{CO}_{2}$, with a first medium change $24-48 \mathrm{~h}$ later. The medium was changed every $2-3$ days. 


\section{Cell metabolic activity measurement}

Synovial fibroblast proliferation was measured using CellTiter $96^{\circ}$ Aqueous One Solution Cell Proliferation Assay (Promega) according to the manufacturer's instructions. Fibroblasts were cultured in 96-well tissue culture plates at $5 \times 10^{3}$ cells/well over $72 \mathrm{~h}$. Cell culture supernatants were aspirated and $100 \mu \mathrm{L}$ of serum-free DMEM medium containing 10\% CellTiter reagent was added to each well. Plates were incubated for $2 \mathrm{~h}$ at $37^{\circ} \mathrm{C}$ and the absorbance was read at $490 \mathrm{~nm}$ using an Infinite F50 plate reader (Tecan, UK). Five replicates of each exposure were tested and the entire assay was repeated in three separate experiments. The cell viability was determined as a percentage of control cell viability.

\section{Collagen contraction}

A contraction assay was used to measure collagen contraction by fibroblasts. Fibroblasts $\left(1 \times 10^{4}\right.$ cells $\left./ \mathrm{ml}\right)$ were first mixed with $1.5 \mathrm{mg} / \mathrm{ml}$ neutralized rat tail collagen solution (3 parts of $2 \mathrm{mg} / \mathrm{ml}$ collagen solution with 1 part DMEM medium). $0.5 \mathrm{ml}$ fibroblast-containing collagen solution was added to 24-well cell culture plates and allowed to polymerize for $30 \mathrm{~min}$ at $37^{\circ} \mathrm{C}$. Then $0.5 \mathrm{ml}$ of serum-free cell culture medium was added and the gels were incubated for $12 \mathrm{~h}$. Following incubation, these gels containing fibroblasts were gently detached from the wells using fine straight and curved forceps. The medium was aspirated and replaced with DMEM cell culture media containing either $10 \%$ serum, PDGF-BB (10 ng/ml, Peprotech, UK) or TGF- $\beta 1$ ( $5 \mathrm{ng} / \mathrm{ml}$, Peprotech, UK). The gel contraction was recorded following $12 \mathrm{~h}$ of exposure to these media.

\section{Mechanical properties measurement Traction force measurement}

Briefly, $22 \mathrm{~mm}$ coverslips (Corning, USA) were treated with Hexamethyldisilazane (HMDS, VWR, UK), rinsed, air dried and a gel solution of 5\% containing acrylamide (Biorad, UK), 0.25\% bis-acrylamide (Biorad, UK), 0.05\% ammonium persulfate (10\% APS, Sigma), $0.1 \% 1$, 2-Bis (dimethylamino) ethane (TEMED, Sigma, UK) and carboxylate-modified beads (fluorescent red, diameter $0.2 \mu \mathrm{m}, 1 \% \mathrm{v} / \mathrm{V}$, ThermoFisher) was applied. After polymerization, the gel was activated with heterobifunctional cross-linker $1 \mathrm{mg} / \mathrm{ml}$ Sulfo-SANPAH (Sigma) and thoroughly rinsed with distilled water. The gels were then coated with type I rat tail collagen $(0.2 \mathrm{mg} / \mathrm{ml})$ overnight. Fibroblasts were plated at a density of $3 \times 10^{4}$ cells/well in a serum-free DMEM and incubated overnight at $37^{\circ} \mathrm{C}$ and $5 \% \mathrm{CO}_{2}$ in a humid incubator before imaging (LS720 Microscope, Lumascope, UK). Brightfield for cells and $525 \mathrm{~nm}$ for bead image stacks were acquired at each position. After imaging, fibroblasts were lysed with $1 \%$ SDS with the positions re-imaged. Five different cells from each of 8 control samples and 8 MoM samples were analysed. A custom-built analysis pipeline implemented in LabVIEW (National Instruments, Austin, TX) was used to measure the traction forces as previously described [13].

\section{Atomic force microscopy (AFM)}

The stiffness of fibroblasts was measured using Advanced Quantitative Imaging mode on a JPK NanoWizard 4 system in combination with an inverted microscope (Axio Observer Z1, Zeiss). Cantilevers (HYDRA6R, AppNano, UK) were calibrated by measuring the sensitivity against a stiff polystyrene substrate, and then fitting the resonance peak in the thermal noise spectrum to determine the spring constant (approximately $0.08 \mathrm{~N} / \mathrm{m}$ ). A ROI of $100 \times 100 \mu \mathrm{m}$ was selected to cover an entire cell. Indentations were performed in a format of $32 \times 32$ at a loading/ unloading speed of $50 \mu \mathrm{m} / \mathrm{s}$, which minimized cell movement during scanning without compromising the resolution. All the AFM experiments were carried out at $37^{\circ} \mathrm{C}$ in FBS-free medium. Force-indentation curves were further processed involving background subtraction, height correction and fitting with a modified Hertzian model.

\section{Collagen production}

To measure the hydroxyproline produced by fibroblasts, the culture medium was collected after $48 \mathrm{~h}$ of culture and hydrolyzed in $6 \mathrm{~N} \mathrm{HCl}$ for $24 \mathrm{~h}$ at $105^{\circ} \mathrm{C}$. The amount of 4-hydroxy proline in the hydrolyzate was determined at a wavelength of $570 \mathrm{~nm}$ on a microplate reader (BMG Nova Star, BMG LABTECH) using the conventional colorimetric method [14].

\section{Chemokine profiling}

The cell culture media was harvested after $48 \mathrm{~h}$ and processed for profiling chemokines using the Proteome Profiler Human Chemokine Array Kit (R\&D system, Minneapolis, MN), which detects a panel of 31 chemokines (Table 2). The array membranes were reacted with the mixture of conditioned media and the antibody cocktail for $18 \mathrm{~h}$ at $4{ }^{\circ} \mathrm{C}$. After 3 washes, they were briefly incubated with secondary antibodies conjugated with horseradish peroxidase (HRP). The membranes were then exposed to HRP substrate for $30 \mathrm{~min}$. The intensity of the reaction was quantified using a BIO-RAD ChemiDoc MP Imaging System (Bio-Rad, UK); the pixel densities on developed X-ray film were analysed using Image Lab software (Bio-Rad, UK). The supernatants were assayed for MCP-1 concentrations with a human MCP-1 ELISA kit (Biolegend, UK).

\section{Transwell migration assay}

Monocyte migration was investigated using $6.5 \mathrm{~mm}$ Transwell chambers with $8 \mu \mathrm{m}$ pores (Costar, Corning, 
Table 2 Chemokines detected with the Proteome Profiler Human Chemokine Array

\begin{tabular}{llll|}
\hline CCL1/I-309 & CCL21/6Ckine & CXCL8/IL-8 & CCL18/PARC \\
\hline CCL2/MCP-1 & CCL22/MDC & CXCL9/MIG & CCL19/MIP-3 beta \\
CCL3/CCL4 & CCL26/Eotaxin-3 & CXCL10/IP-10 & CCL20/MIP-3 alpha \\
CCL5/RANTES & CCL28 & CXCL11/I-TAC & CXCL5/ENA-78 \\
\hline CCL7/MCP-3 & Chemerin & CXCL12/SDF-1 & CXCL7/NAP-2 \\
\hline CCL14/HCC-1 & CX3CL1/Fractalkine & CXCL16 & Midkine \\
\hline CCL15/MIP-1 delta & CXCL1/GRO alpha & CXCL17/VCC-1 & XCL1/Lymphotactin \\
\hline CCL17/TARC & CXCL4/PF4 & IL-16 & \\
\hline
\end{tabular}

NY, USA). Briefly, $1 \times 10^{5}$ U937 monocytes in serumfree DMEM medium were added to the upper chamber of an insert. The lower chamber was filled with cell culture supernatant from fibroblasts cultured for $48 \mathrm{~h}$ to encourage cell migration down the FBS chemotactic gradient. After $12 \mathrm{~h}$, cells that transmigrated to the bottom well were collected and counted.

\section{Fluorescence-activated cell sorting (FACS) analysis}

After $24 \mathrm{~h}$ of serum withdrawal, the percentage of apoptotic fibroblast were determined via FACS utilising a FITC Annexin V Apoptosis Detection Kit with PI (Biolegend, UK). The collected cell suspensions were transferred to FACS tubes (BD Biosciences; Oxford, UK), centrifuged for $5 \mathrm{~min}$ at $350 \mathrm{xg}$ and the supernatant aspirated. Cell suspensions were washed twice with cold cell staining buffer (Biolegend, UK), and then resuspended in Annexin V Binding Buffer at a concentration of $0.5 \times 10^{7}$ cells $/ \mathrm{ml}$. $100 \mu \mathrm{l}$ of cell suspension was transferred to a $5 \mathrm{ml}$ test tube and $5 \mu \mathrm{l}$ of FITC Annexin V with $10 \mu \mathrm{l}$ of Propidium Iodide Solution was added. The mixture of cells and staining reagent was allowed to incubate for $15 \mathrm{~min}$ at room temperature in the dark prior to analysis with flow cytometry. Stained cells were washed and re-suspended in $200 \mu \mathrm{l}$ of PBS and analysed using a BD ACCURI C 6 flow cytometer (Biosciences, USA) and FlowJo software (TreeStar, USA).

\section{Co-culture of U937 monocytes and synovial fibroblasts}

Porous plasma-treated polycarbonate inserts (pore size: $0.4 \mu \mathrm{m}$, Transwell, Corning) were used for non-contact co-culture of fibroblasts and U937 monocytes. MoM and control synovial fibroblasts were added to the top of the Transwell $^{\circ}$ insert at $2.5 \times 10^{4}$ cells/well and U937 monocytes were cultured at $5 \times 10^{4}$ cells/well in 24-well tissue culture plates providing a co-culture within each well. The increase in the differentiation of U937 monocytes was quantified by counting the cells that floating in the medium collected from each well of tissue culture plates by $0 \mathrm{~h}, 12 \mathrm{~h}$ and $24 \mathrm{~h}$ of co-culture.

\section{Image analysis}

Images were acquired on an upright microscope (Nikon Eclipse). For image quantification, the mean percentage area positive for 10 randomly selected high powered fields ( $\times 10$ magnification) was calculated using ImageJ analysis software as described previously [15].

\section{Data analysis and statistics}

All data were expressed as the mean + SEM of at least three independent experiments. The statistical differences between groups were analysed with Student t-test (SPSS Inc., Chicago, IL, USA). A value of $p<0.05$ was considered statistically significant.

\section{Results}

Tissue remodelling of patients with metal-on-metal hip implants

A gross morphological change to the periprosthetic tissue was observed in tissues collected from patients with failed MoM implants. The biopsies were generally denser, pigmented and contained fibrotic regions (Fig. 1a). At the microscopic level metal wear or corrosion products in some of the MoM cases were observed, which appeared a dark-grey or green morphology (Fig. 1a) as previously reported [16]. To determine the level of a fibrotic change, we assessed the collagen deposition. The control tissues obtained at primary THA operations were characterised by a wide distribution of fat cells (adipocytes) and approximately $20 \%$ collagen content as determined by Picrosirius Red staining (Fig. 1b,e) and more specifically collagen type I (Fig. 1c,f). Elevated levels of collagen were observed in the MoM tissues, with approximately $55 \%$ positive staining for collagen and $45 \%$ for collagen type I. We further observed a significant increase in the number of myofibroblasts in the MoM tissues from less than $4 \%$ to nearly $9 \%$ (Fig. 1d,g) as determined by the positive staining for $\alpha$ -SMA.

\section{MoM fibroblast are more resistant to apoptosis}

The balance between cell proliferation and apoptosis plays a key role in tissue homeostasis. First, we investigated whether the increased matrix deposition in tissue 


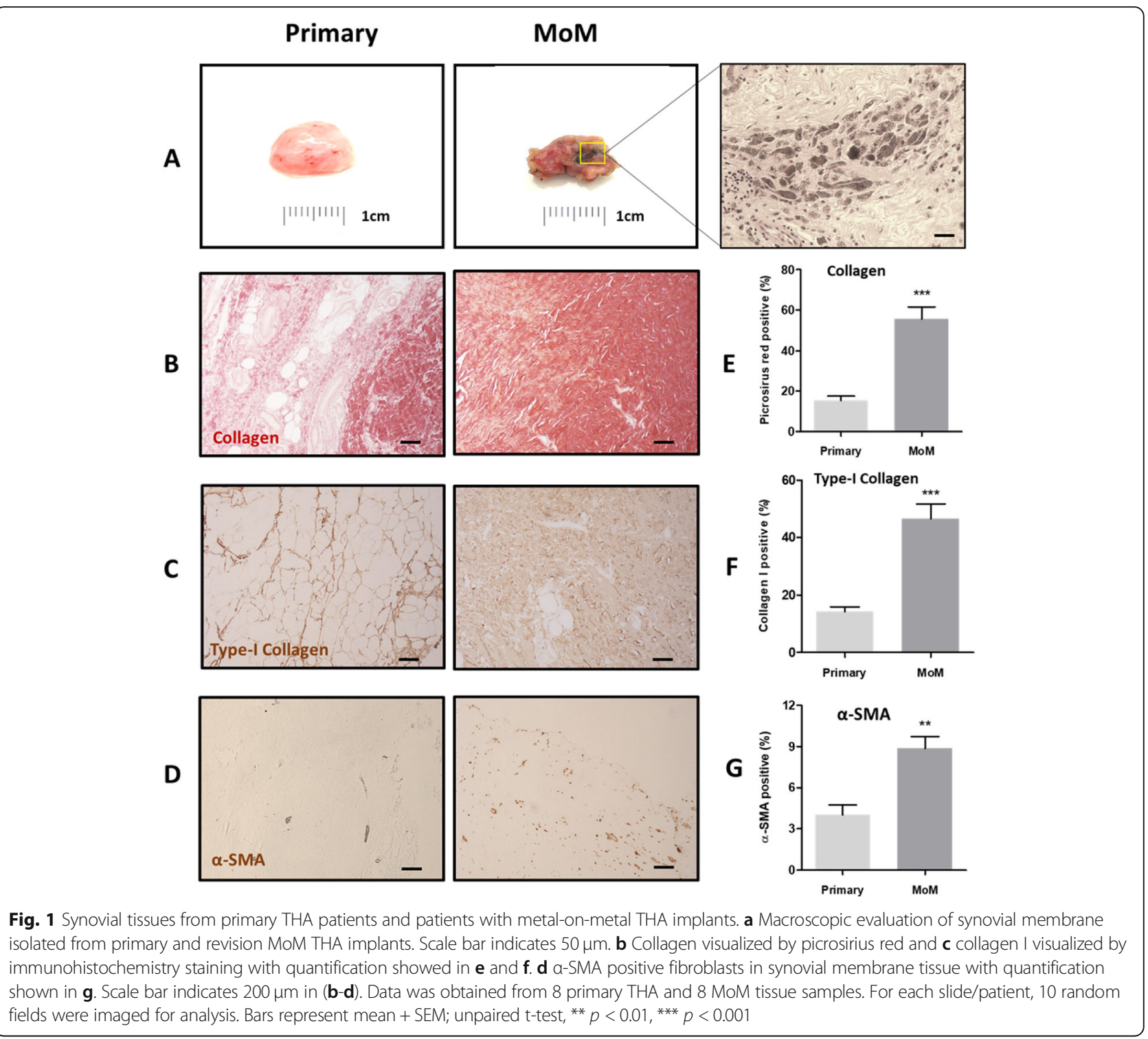

is due to an increase in proliferative capacity of synovial fibroblasts exposed to metal products from MoM implants. We found that ex vivo MoM fibroblasts showed the same proliferative capacity as control synovial fibroblasts over $72 \mathrm{~h}$ of culture (Fig. 2a). It has been reported that fibroblast survival could be altered under certain pathological condition in a manner of reduced cell apoptosis, which contributes to the development and maintenance of fibrosis [17]. Although no significant difference in the proliferative ability was identified between primary and MoM fibroblasts, MoM synovial fibroblasts showed reduced apoptosis rates following serum withdrawal (Fig. 2b) compared with the control cells. Primary (Fig. 2c) and MoM fibroblast (Fig. 2d) survival was determined by serum withdrawal-induced apoptosis, analysed by an Annexin V-FITC/PI assay via FACS.
According to the results shown, after $24 \mathrm{~h}$ of serum withdrawal, the percentage of apoptotic cells for MoM fibroblasts were approximately $6 \%$, compared to nearly $14 \%$ for the control fibroblasts. These data suggest that MoM fibroblasts are more resistant to apoptosis, which may partially explain their accumulation and tissue remodelling in the patients with CoCr alloy implants.

\section{Matrix remodelling by human synovial fibroblasts}

To elucidate the in vivo tissue alterations in tissue density and matrix deposition, the isolated synovial fibroblasts were examined ex vivo for matrix production. We measured the hydroxyproline content as a surrogate for collagen. A statistically significant increase in production of pro-collagen by MoM synovial fibroblasts was observed when compared to the control synovial 
A

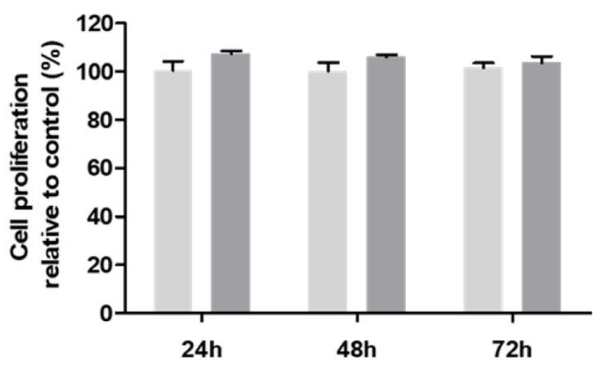

C

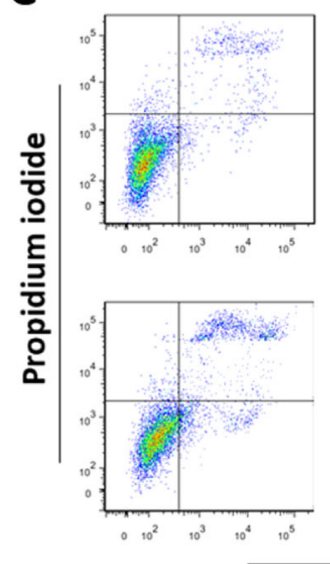

D
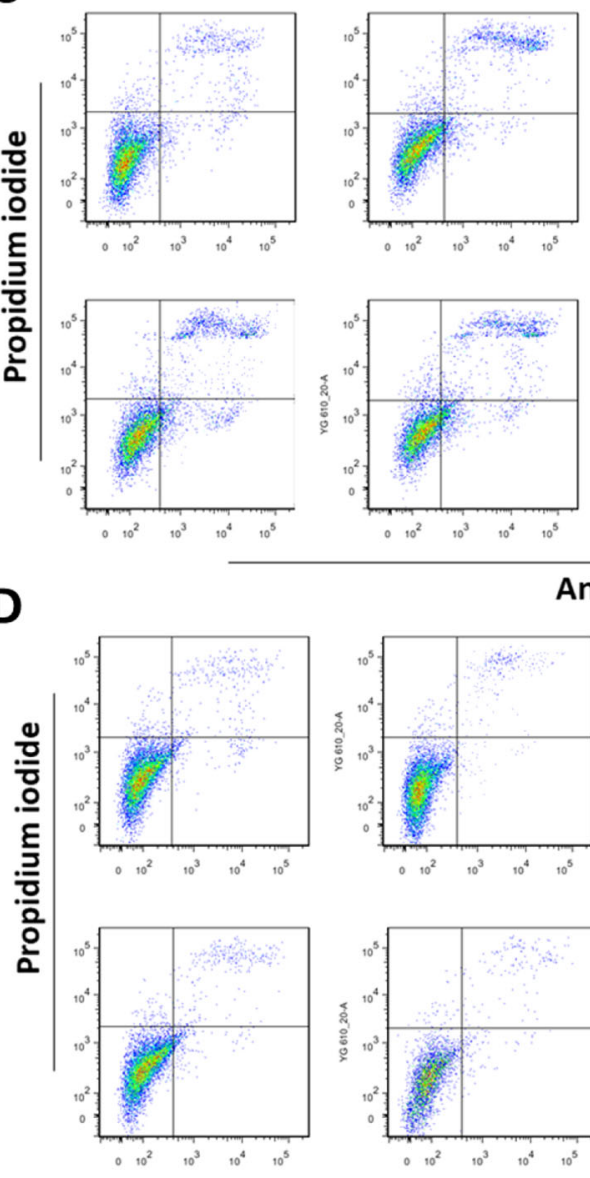

B

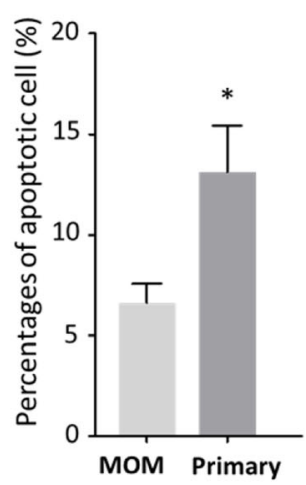

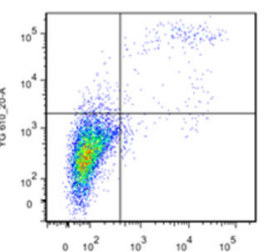
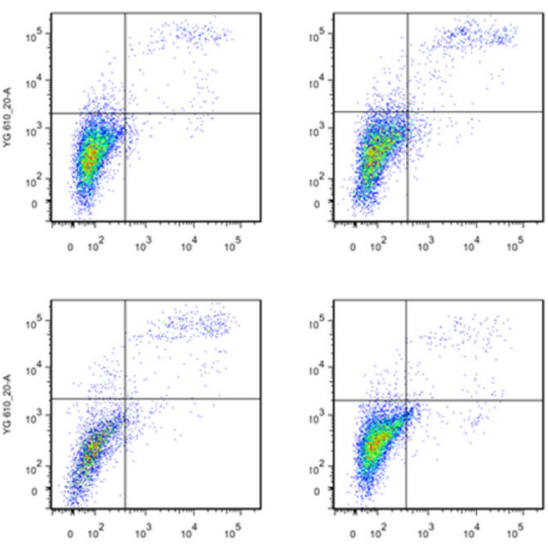

Annexin V
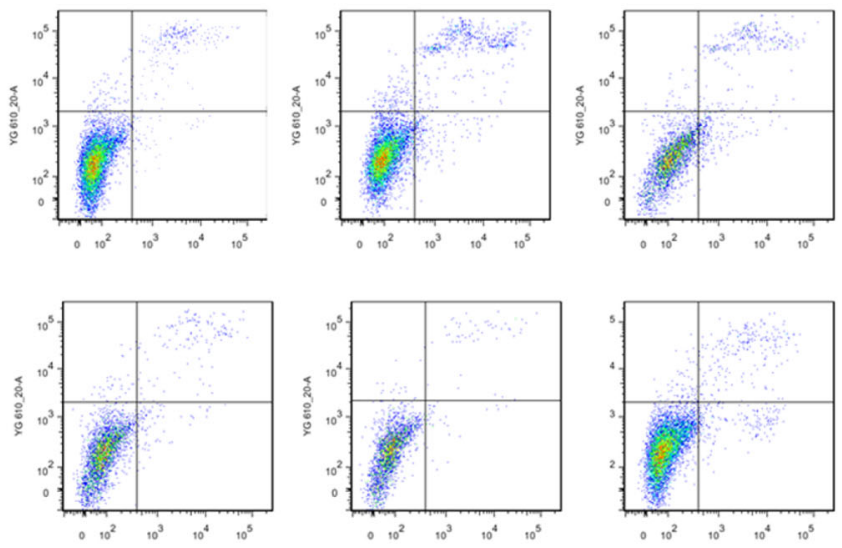

Annexin V

Fig. 2 Proliferation and apoptosis of primary and MoM fibroblast. a Proliferation of primary and MoM fibroblasts over $72 \mathrm{~h}$ measured by MTS assay. Apoptosis of primary and MoM fibroblast induced by serum withdrawal. b Quantification of apoptotic cells of primary and MoM fibroblasts. Data was from 3 independent experiments using fibroblasts from each primary and MOM patient; unpaired Student's t-test; * $p<0.05$. Representative cell apoptosis results of primary (c) and MoM (d) fibroblasts from each patient assessed by Annexin V-FITC/PI assay via FACS

fibroblasts from primary THAs (Fig. 3a). This suggests that synovial fibroblasts isolated from tissues surrounding a MoM implant which requires revision, but has been in use, releasing metal and ionic debris for 5-14 years, have enhanced matrix production ability and retain that capability ex vivo in the absence of the metal stimulation. To investigate whether the MoM synovial fibroblasts have greater capability to deform matrix, which is an important aspect of pro-fibrotic alteration, their contractility potential was analysed. An in vitro 
A

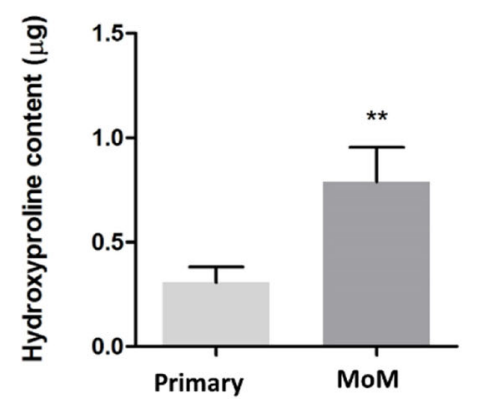

C

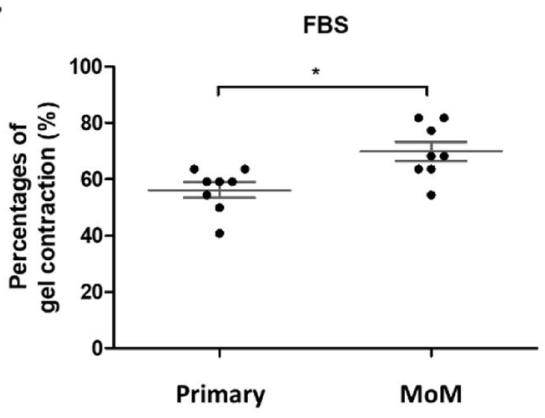

E

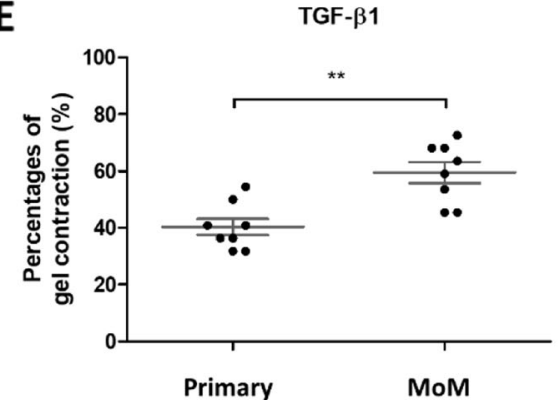

B
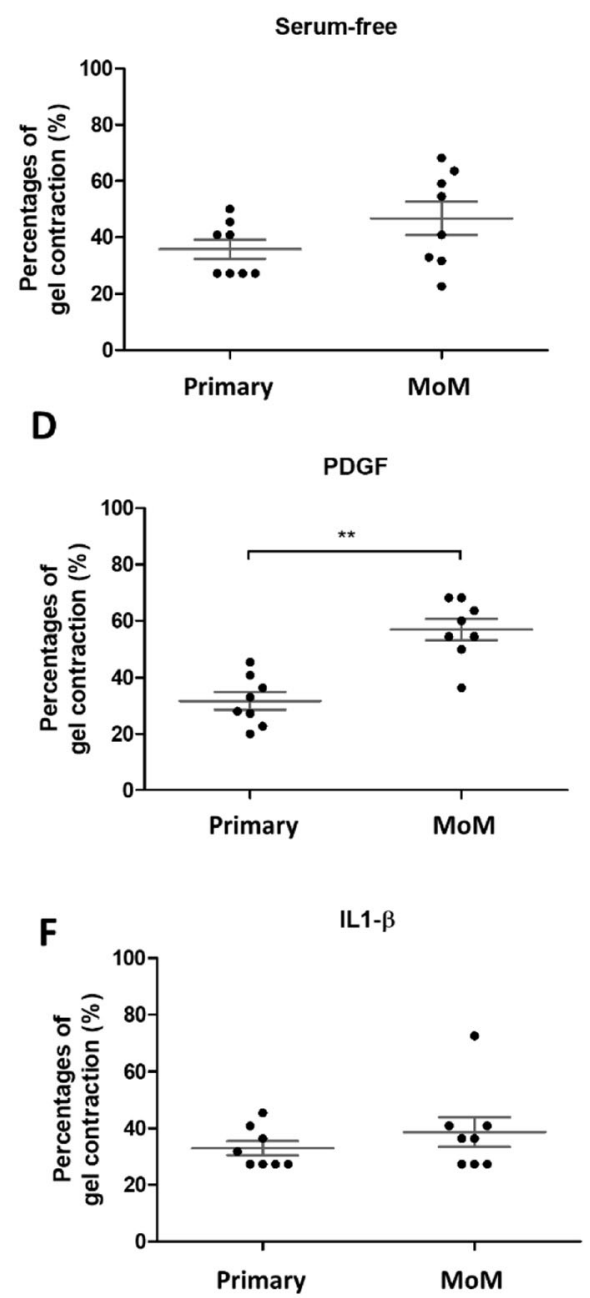

Fig. 3 Synovial fibroblasts from patients with MoM implant display increased ECM matrix production and contractility. a Collagen released by cultured synovial fibroblasts into the cell culture medium after $48 \mathrm{~h}$. Bars represent mean + SEM. b-f Fibroblasts from MoM and primary THA were embedded in a collagen matrix and contraction was measured after $48 \mathrm{~h}$, in serum-free medium (b), medium with $10 \%$ serum (c) or with cytokines $(10 \mu \mathrm{g} / \mathrm{ml}$ PDGF (d), $5 \mathrm{\mu g} / \mathrm{ml} \mathrm{TGF- \beta 1}$ (e) and $10 \mathrm{ng} / \mathrm{mL} \mathrm{IL-1 \beta}$ (f). Shown is mean \pm SEM for gel contraction after $48 \mathrm{~h}$ of culture. Each individual data point is the average of 3 independent experiments using fibroblasts from each patient. ${ }^{*} p<0.05,{ }^{* *} p<0.01$

collagen gel contraction assay was performed in various conditions, including serum-free conditions and in the presence of serum (10\% FBS), PDGF (10 ng/mL), TGF$\beta 1(5 \mathrm{ng} / \mathrm{mL})$ and $\mathrm{IL}-1 \beta(10 \mathrm{ng} / \mathrm{mL})$. In the serum-free condition, the contractility of MoM and control synovial fibroblasts was comparable (Fig. 3b), while in the presence of serum the contractility of MoM synovial fibroblasts was significantly increased $(p<0.05)$. When mimicking inflammatory conditions, by adding PDGF or TGF $\beta$, the MoM fibroblast contractility further increased to $56.9 \pm 3.8 \%$ (from $31.7 \pm 3.1 \%$ ) in the presence of PDGF $(p<0.01)$ and to $59.5 \pm 3.7 \%$ (from $40.3 \pm 2.9 \%$ ) in the presence of TGF- $\beta(\mathrm{p}<0.01)$ (Fig. $2 \mathrm{c}-\mathrm{e})$. While IL-1 $\beta$ have not obvious effects on the contractility of both MoM and control synovial fibroblasts. These results suggest that MoM synovial fibroblasts displayed a distinctive bio-mechanical response to associated inflammatory signalling.

\section{MoM-induced alterations of fibroblast mechanical properties}

The contractility of cells is regulated by their mechanical properties. Previously, we showed that cobalt exposure affects cell elastic modulus and contractile force of human dermal fibroblasts [12]. To study the link between the increased contractility of MoM synovial fibroblasts and alterations in their mechanical properties, we performed Traction Force Microscopy and Atomic Force Microscopy. First, we measured the traction forces of resting synovial fibroblast (following $24 \mathrm{~h}$ of serum 
starvation). A significant increase, of nearly $60 \%$, in cellular traction forces $(p<0.05)$ was observed in MoM synovial fibroblasts when compared to the control (Fig. 4a and b). Cell stiffness is closely associated with cell behaviour and the alterations in cell traction forces could be coupled with alterations in cell elastic modulus, which has been suggested as an indicator of cytoskeleton rearrangement [18]. We observed that the cell elastic modulus measured with AFM (Fig. 4c and d) was also significantly higher $(3.33 \pm 0.65 \mathrm{KPa})$ in MoM synovial fibroblasts compared with the control $(2.18 \pm 0.74 \mathrm{KPa})$ as shown in Fig. 4e.

\section{MoM fibroblasts promote immune cell invasion and differentiation}

As reported in the previous section, growth factors released by immune cells drive the contractile activity of MoM synovial fibroblasts. One of the key immune cells activated by cobalt released from $\mathrm{CoCr}$ implants (MoM) are $\mathrm{CD} 8^{+}$macrophages, which release pro-fibrotic signals and enhance the fibrotic response of fibroblasts [4]. The histological analysis of the tissue biopsies showed a significant increase in $\mathrm{CD}^{+} 8^{+}$macrophages in MoM tissues (Fig. 5a). However, whether the MoM synovial fibroblast release pro-inflammatory signals to recruit macrophages, is unclear. To study the pro-inflammatory properties of MoM synovial fibroblasts, a migration assay was performed. The U937 monocyte migration was significantly enhanced in the presence of cell culture supernatants from MoM fibroblasts (Fig. 5b). By examining the chemokine profile of the supernatants (Fig. 5c), a significant elevation in MCP-1 (monocyte chemoattractant protein 1) was observed in the MoM fibroblast cell culture supernatant compared to the control (Fig. $5 \mathrm{~d} \& \mathrm{e})$. This suggests that MoM fibroblasts could potentiate the tissue immune response through the

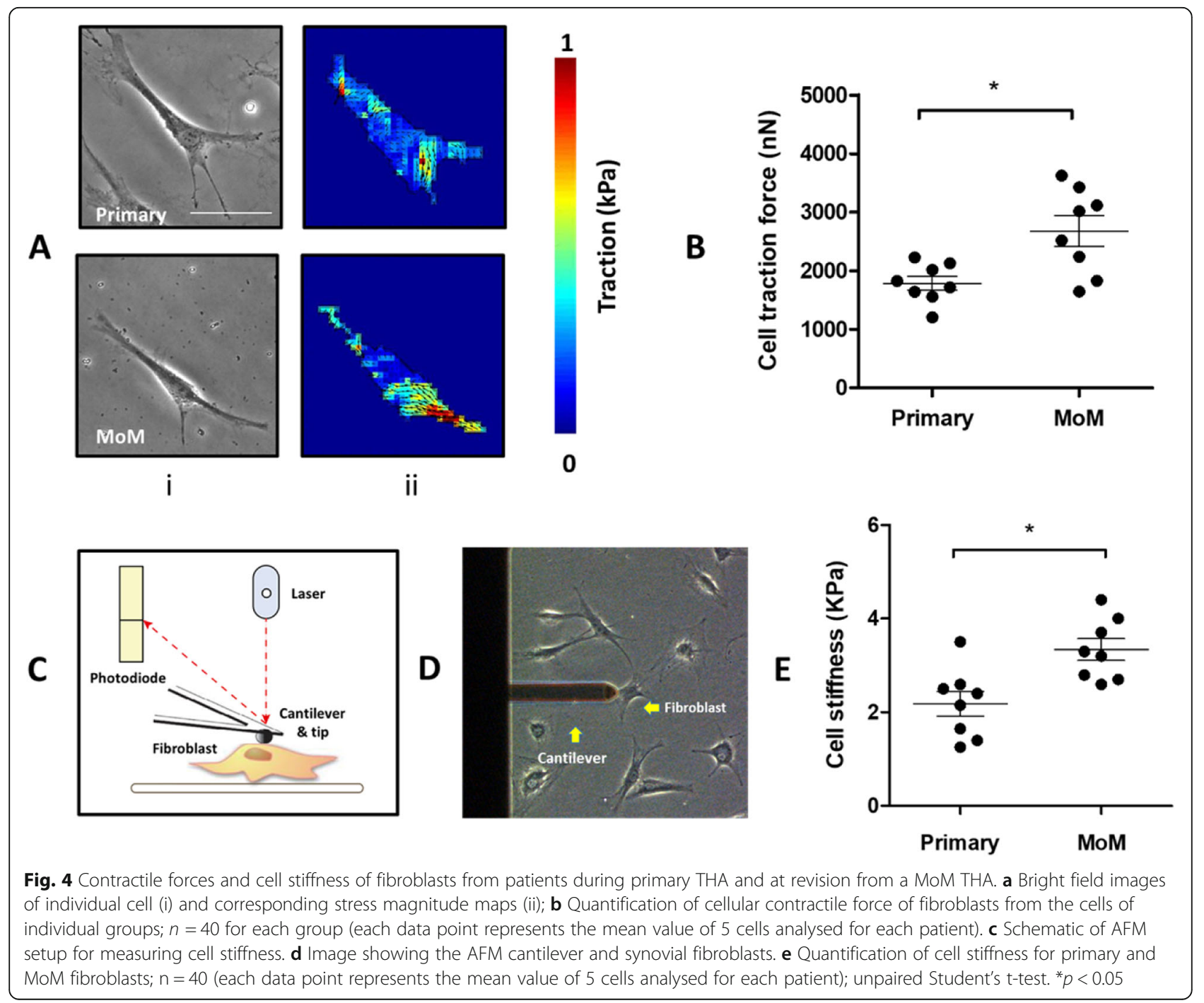




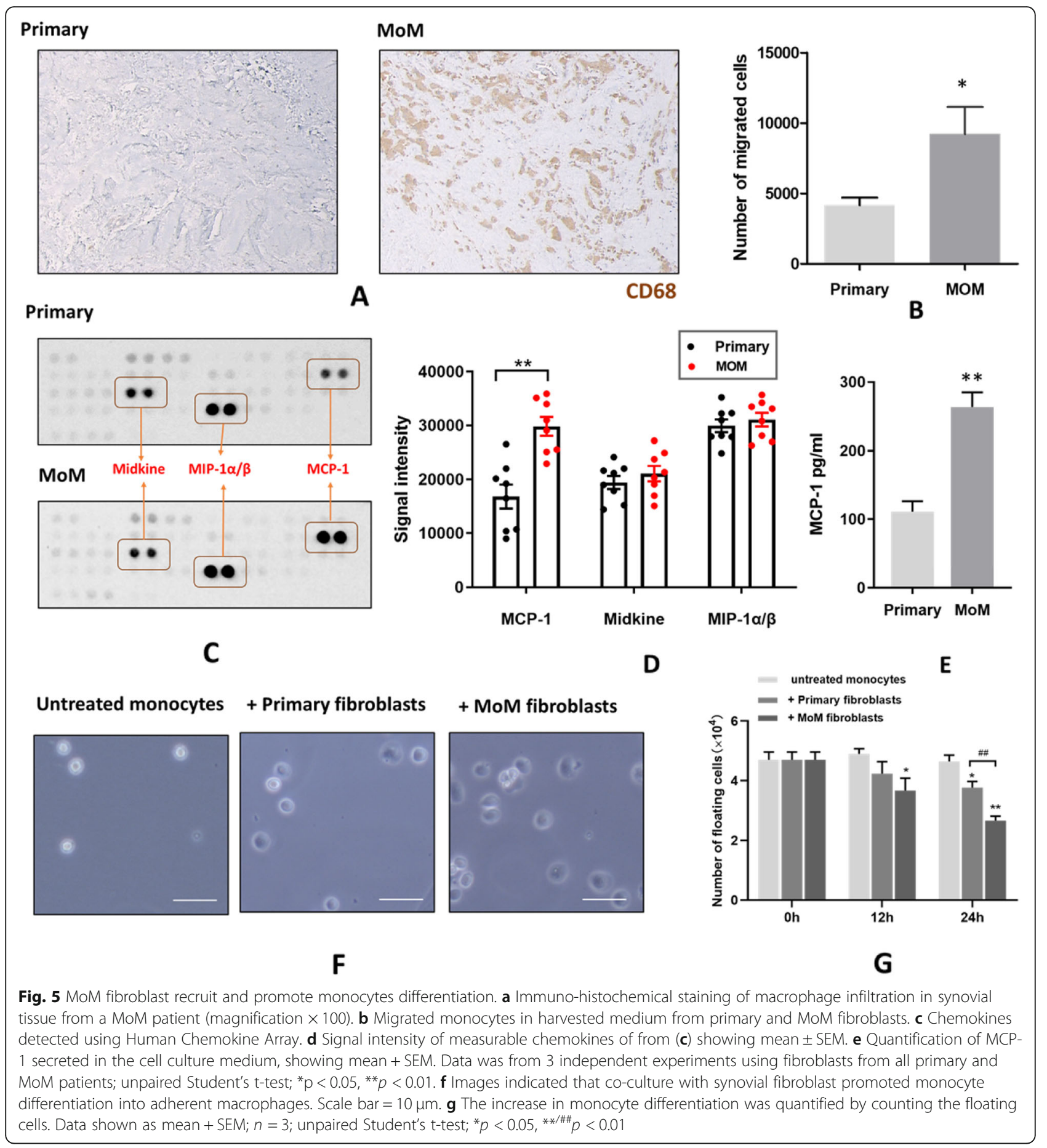

secretion of MCP-1, thereby enhancing monocyte infiltration. Also, to test the effect of MoM fibroblasts on monocyte differentiation, fibroblasts from MoM and control patients were co-cultured with U937 human monocytes. It could be noticed that both MoM and control synovial fibroblasts induced monocyte differentiation into macrophages, as indicated by increased cellular adhesion to the substrate with the number of floating U937 monocytes were significantly lower compared to the untreated cells at the $24 \mathrm{~h}$ time points (Fig. $5 \mathrm{~g})$. Furthermore, the number of floating U937 monocytes decreased to a greater extent in the presence of MoM synovial fibroblasts compared with control fibroblasts (Fig. 5g). Therefore, these results indicated the 
presence of higher amount of pro-inflammatory factors from MoM fibroblasts that could promote the invasion and differentiation of human monocyte.

\section{Discussion}

Despite being one of the most significant issues in the field of total hip arthroplasty, the precise mechanisms associated with adverse tissue reactions to wear debris generated in patients following the implantation of a MoM implant (which generates $\mathrm{CoCr}$ particles and dissociated ions in use) responsible for the devastating failure of these implants, remains poorly understood. Many cell lines have previously been used to investigate the effects of $\mathrm{CoCr}$ wear particles and ions in vitro including macrophages, lymphocytes and osteoblasts. In vitro studies have revealed that $\mathrm{CoCr}$ metallic debris and ions trigger the release of an array of proinflammatory mediators from macrophages [19-21], such as transforming growth factor $\alpha$ (TGF- $\alpha)$, macrophage colonystimulating factor (M-CSF), platelet-derived growth factor (PDGF) and epidermal growth factor (EGF). Cobalt has also been known to promote intracellular reactive oxygen species formation that lead to a wide range of deleterious effects and irreversible damage.

Fibroblasts are one of the central participants for wound healing and inflammation. They play a positive role in promoting the recovery of functional tissue but equally are capable of driving it towards chronic inflammation, fibrosis or even tissue necrosis depending on their exposure to biochemical cues [19]. It has also been reported that fibroblasts, one of the most active tissue resident mesenchymal cells, may display site or diseasespecific phenotypes after exposure to inflammatory signals, which could induce a dysregulated homeostatic balance of extracellular environment [22]. This motivated the present study, in which in vitro assays were applied to characterise some of the key features of primary human synovial fibroblasts and investigate their contribution to the tissue response and disease progression.

The findings of this study demonstrated that synovial tissues from patients undergoing revision surgery due to the failure of the MoM device were macroscopically and histologically different from tissues isolated from patients undergoing primary THA surgery, with evidence of extensive tissue remodelling characterised by replacement of adipocytes with dense areas of collagen (Fig. 1ac). In addition, we observed enhanced activation of synovial fibroblast activation with a significant increase in the number of myofibroblasts in the tissues (Fig. 1d). Fibroblasts are the primary cell type responsible for ECM proteins synthesis to build up the connective tissue. Moreover, they promote ECM remodelling via transmitting force to contract and compact the surrounding matrices to achieve tensional homoeostasis, which have a significant impact on other cells' behaviour [23]. Our data demonstrated that stimulated fibroblasts isolated from patients undergoing a MoM THA revision were more efficient at contracting collagen matrix and were also more responsive to biochemical stimuli involving cytokines and growth factors (Fig. 3). They were also found to display altered cell force responses compared to the control primary cells (Fig. 4). Disruption of the balance between cell proliferation and apoptosis could affect tissue homeostasis. The enhanced survival of MoM fibroblasts when exposed to apoptotic signal (Fig. 2) also confirmed the alteration of fibroblasts phenotype and undesired cellular response after exposure to $\mathrm{CoCr}$ metal wear and corrosion products.

Previous research has largely focused on the cytotoxic effects of $\mathrm{CoCr}$ alloy products on fibroblasts ex vivo, with little attention being paid to their influence on local tissue homeostasis in vivo [24-26]. Indeed recent evidence has suggested that the deposition of ECM, produced by fibroblasts as well as tissue mechanics play a vital role in the regulation of the inflammatory process [27]. For instance, stiffer tissue substrates may enhance immune cell invasion and the release of proinflammatory cytokines by macrophages [28, 29]. In addition to their structural role in shaping the microenvironment, fibroblasts also modulate immune responses via their production of cytokines and chemokines [23].

Periprosthetic synovial tissues from patients with MoM THA implants at the revision operation, were characterized by intensive immune cells infiltration. However, the key stimulus of driving the immune cell invasion is largely unknown. MCP-1/CCL2 is one of the critical chemokines that mediates migration and infiltration of monocytes and macrophages during inflammatory response. In vivo and in vitro studies suggest that MCP-1 plays a direct role in the development of many fibrotic lesion by affecting fibroblasts behaviour [30-32]. In this study, we found that fibroblasts surrounding failed MoM THA revision patients may play an indispensable role in the recruitment of immune cells by secreting MCP-1, which enhances chemotactic migration of monocytes and exacerbate inflammatory reactions (Fig. 5). Our results also indicated that MoM fibroblasts produce stimulating factors to promote the differentiation of monocytes, thus potentiating the pathogenic persistence and retention of macrophages in the synovial tissues surrounding MoM implants. Recent studies have reported non-MoM THAs, such as metal-onpolyethylene $(\mathrm{MoP})$, to be presenting adverse tissue responses similar to the MoM THAs. An adverse response to MoP implants, such as the formation of pseudotumours, may still be linked to the release of cobalt ions from the metal head [33-35], albeit at a lower level. This 


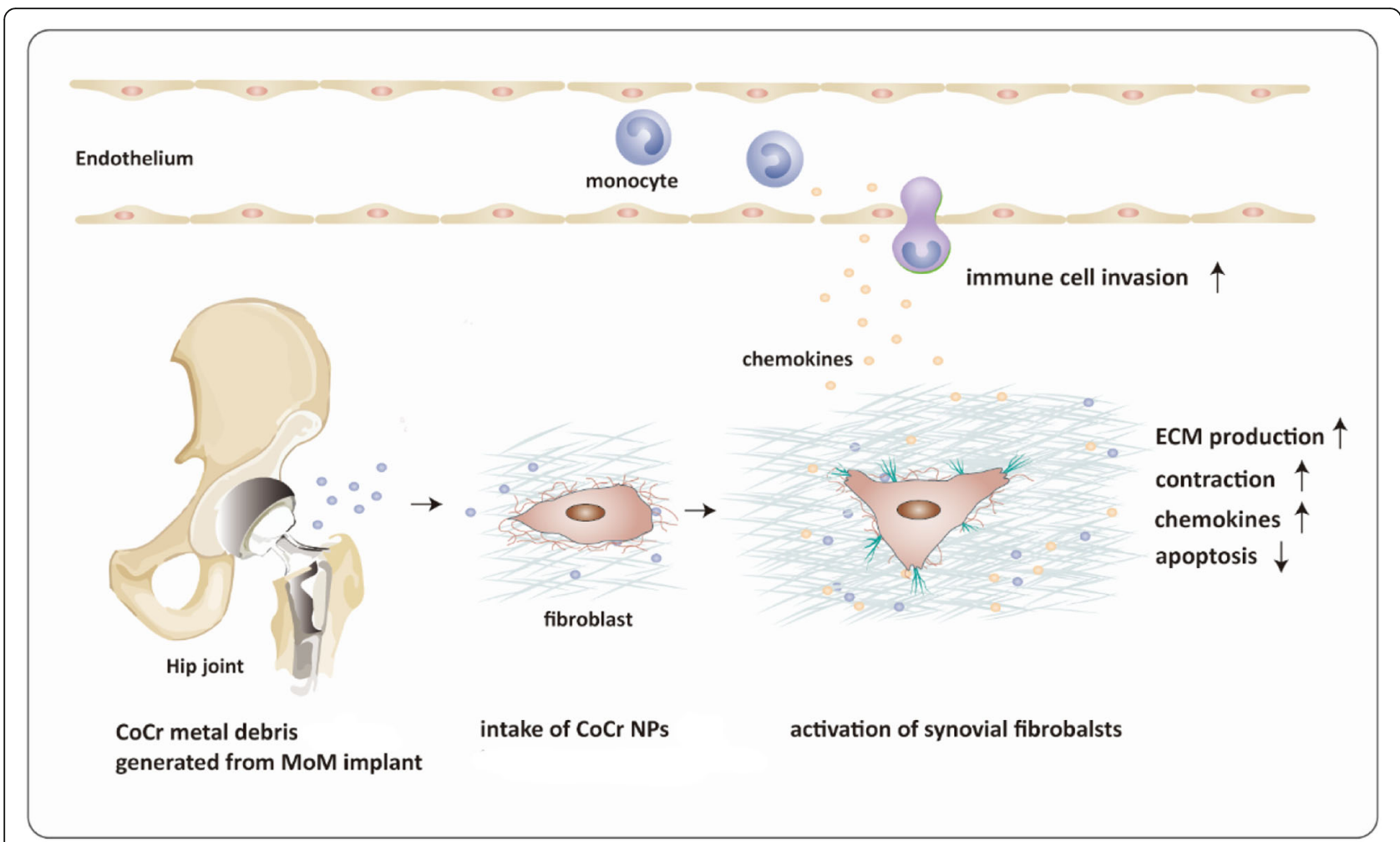

Fig. 6 Schema for the distinctive effects of CoCr particles on fibroblast phenotype and their interplay with immune cells in the patients with CoCr based implants

suggests that any wear/corrosion debris containing cobalt (nano) particulates or ions, may have a detrimental influence on the hip tissue microenvironment.

Overall, we have shown that synovial fibroblasts exposed to MoM THAs in vivo which require revision, undergo phenotypic alteration which is associated with dramatic functional changes that can be observed ex vivo. The response to an inflammatory lesion involves a complex interplay of diverse cellular and tissue elements that restrain tissue invasion and ultimately establish normal tissue integrity [36]. Instead of being a passive player in the immune system, fibroblasts actively define the organisation of tissue microenvironments and modulate cell activities by conditioning the local cellular and cytokine microenvironment [22,37]. The inappropriate activation and accumulation of fibroblasts stimulated by wear debris from failing MoM implants could prevent the resolution of acute inflammation thereby leading to chronic, persistent inflammation.

\section{Conclusions}

This study unravels the distinctive influence of MoM total hip arthroplasties, which release $\mathrm{CoCr}$ wear and corrosion debris in the form of particles and related ions, on the fibroblast phenotype and their interplay with immune cells (Fig. 6). Synovial fibroblasts exposed to MoM THAs in vivo displayed dramatic phenotypic alteration and functional changes. These findings suggest direct in vivo impact of failing MoM THAs on the synovial fibroblasts and local tissue homeostasis.

\section{Acknowledgements}

Authors would like to thank surgeons, nurses and operating room who helped to obtain the tissue samples. The authors are grateful to the China Scholarship Council (CSC) for their support of Jing Xu.

\section{Authors' contributions}

$J X, A N$ and $A H$ designed the project; JX, JY, JC, XZ and YW performed the experiments; JX, JY and AN analyzed data and wrote the paper; AN and JS supervised the entire research project. All authors read and approved the final manuscript.

\section{Funding}

This work was supported in part by the National Natural Science Foundation of China (Grant Nos. 81902275).

\section{Availability of data and materials}

Data supporting the findings are found within the manuscript and supplemental material.

\section{Ethics approval}

Ethics approval [07/Q0401/25, West London Research Ethics Committee] and written patient consent was obtained for the use of tissue samples (synovial membranes) removed during surgery.

Consent for publication

All authors consent for publication. 


\section{Competing interests}

The authors declare that they have no competing interests.

\section{Author details}

'Department of Paediatric Orthopaedics, Xinhua Hospital, School of Medicine, Shanghai Jiao Tong University, Shanghai 200092, China. ${ }^{2}$ Institute of Bioengineering, School of Engineering and Materials Science, Queen Mary University of London, London, UK. ${ }^{3}$ Cardiovascular Division, Faculty of Life Science and Medicine, King's College London, London SE5 9NU, UK. ${ }^{4}$ Department of Spine Surgery, Xinhua Hospital, School of Medicine, Shanghai Jiao Tong University, Shanghai 200092, China. ${ }^{5}$ Institute of Orthopaedics \& Musculoskeletal Science, Royal National Orthopaedic Hospital, University College London, Stanmore HA7 4AP, UK. ' Research Department of Surgical Biotechnology, Division of Surgery and Interventional Sciences, University College London, London NW3 2OG, UK. ${ }^{7}$ Current affiliation: MRC LMB, Cambridge Biomedical Campus, Francis Crick Avenue, Cambridge $\mathrm{CB} 2 \mathrm{OQH}$, UK.

Received: 16 April 2020 Accepted: 12 August 2020

Published online: 27 August 2020

\section{References}

1. Matharu GS, Pandit HG, Murray DW, Judge A. Adverse reactions to metal debris occur with all types of hip replacement not just metal-on-metal hips: a retrospective observational study of 3340 revisions for adverse reactions to metal debris from the National Joint Registry for England, Wales, Northern Ireland and the Isle of Man. BMC Musculoskelet Disord. 2016;17(1):495.

2. Bosker B, Ettema H, Boomsma M, Kollen B, Maas M, Verheyen C. High incidence of pseudotumour formation after large-diameter metal-on-metal total hip replacement: a prospective cohort study. J bone Joint Surg Br. 2012;94(6):755-61.

3. Mahendra G, Pandit H, Kliskey K, Murray D, Gill HS, Athanasou N. Necrotic and inflammatory changes in metal-on-metal resurfacing hip arthroplasties: relation to implant failure and pseudotumor formation. Acta Orthop. 2009; 80(6):653-9.

4. Natu S, Sidaginamale RP, Gandhi J, Langton DJ, Nargol AV. Adverse reactions to metal debris: histopathological features of periprosthetic soft tissue reactions seen in association with failed metal on metal hip arthroplasties. J Clin Pathol. 2012:65(5):409-18.

5. Doorn PF, Campbell PA, Worrall J, Benya PD, McKellop HA, Amstutz HC. Metal wear particle characterization from metal on metal total hip replacements: transmission electron microscopy study of periprosthetic tissues and isolated particles. J Biomed Mater Res. 1998;42(1):103-11.

6. Billi F, Campbell P. Nanotoxicology of metal wear particles in total joint arthroplasty: a review of current concepts. J Appl Biomaterials Biomechanics. 2010;8(1):1-6.

7. Xia Z, Ricciardi BF, Liu Z, von Ruhland C, Ward M, Lord A, et al. Nanoanalyses of wear particles from metal-on-metal and non-metal-on-metal dual modular neck hip arthroplasty. Nanomedicine. 2017;13(3):1205-17.

8. Campbell P, Ebramzadeh E, Nelson S, Takamura K, De Smet K, Amstutz HC. Histological features of pseudotumor-like tissues from metal-on-metal hips. Clin Orthopaedics Related Res. 2010;468(9):2321-7.

9. Willert H-G, Buchhorn GH, Fayyazi A, Flury R, Windler M, Köster G, et al. Metal-on-metal bearings and hypersensitivity in patients with artificial hip joints: a clinical and histomorphological study. JBJS. 2005;87(1):28-36.

10. Athanasou N. The pathobiology and pathology of aseptic implant failure. Bone Joint Res. 2016;5(5):162-8.

11. Dakin SG, Buckley CD, Al-Mossawi MH, Hedley R, Martinez FO, Wheway K, et al. Persistent stromal fibroblast activation is present in chronic tendinopathy. Arthritis Res Ther. 2017;19(1):16.

12. Xu J, Nyga A, Li W, Zhang X, Gavara N, Knight M, et al. Cobalt ions stimulate a fibrotic response through matrix remodelling, fibroblast contraction and release of pro-fibrotic signals from macrophages. European Cells Materials. 2018;36:142-55.

13. Gavara N, Sunyer R, Roca-Cusachs P, Farre R, Rotger M, Navajas D. Thrombin-induced contraction in alveolar epithelial cells probed by traction microscopy. J Appl Physiol (1985). 2006;101(2):512-20.

14. Reddy GK, Enwemeka CS. A simplified method for the analysis of hydroxyproline in biological tissues. Clin Biochem. 1996;29(3):225-9.

15. Jensen EC. Quantitative analysis of histological staining and fluorescence using ImageJ. Anat Rec. 2013;296(3):378-81.
16. Nyga A, Hart A, Tetley TD. Molecular analysis of HIF activation as a potential biomarker for adverse reaction to metal debris (ARMD) in tissue and blood samples. J Biomed Mater Res B Appl Biomater. 2019;107(5):1352-62.

17. Liu X, Das AM, Seideman J, Griswold D, Afuh CN, Kobayashi T, et al. The CC chemokine ligand 2 (CCL2) mediates fibroblast survival through IL-6. Am J Respir Cell Mol Biol. 2007;37(1):121-8.

18. Fletcher DA, Mullins RD. Cell mechanics and the cytoskeleton. Nature. 2010; 463(7280):485.

19. Man K, Jiang LH, Foster R, Yang XB. Immunological Responses to Total Hip Arthroplasty. J Functional Biomaterials. 2017;8(3).

20. Posada OM, Tate RJ, Grant MH. Effects of $\mathrm{CoCr}$ metal wear debris generated from metal-on-metal hip implants and co ions on human monocyte-like U937 cells. Toxicol vitro. 2015;29(2):271-80.

21. Kaufman AM, Alabre Cl, Rubash HE, Shanbhag AS. Human macrophage response to UHMWPE, TiAIV, $\mathrm{CoCr}$, and alumina particles: analysis of multiple cytokines using protein arrays. J Biomed Mater Res A. 2008;84(2): 464-74.

22. Buckley CD, Pilling D, Lord JM, Akbar AN, Scheel-Toellner D, Salmon M. Fibroblasts regulate the switch from acute resolving to chronic persistent inflammation. Trends Immunol. 2001;22(4):199-204.

23. Van Linthout S, Miteva K, Tschöpe C. Crosstalk between fibroblasts and inflammatory cells. Cardiovasc Res. 2014;102(2):258-69.

24. Papageorgiou I, Brown C, Schins R, Singh S, Newson R, Davis S, et al. The effect of nano- and micron-sized particles of cobalt-chromium alloy on human fibroblasts in vitro. Biomaterials. 2007;28(19):2946-58.

25. Behl B, Papageorgiou I, Brown C, Hall R, Tipper JL, Fisher J, et al. Biological effects of cobalt-chromium nanoparticles and ions on dural fibroblasts and dural epithelial cells. Biomaterials. 2013;34(14):3547-58.

26. Smith LJ, Holmes AL, Kandpal SK, Mason MD, Zheng T, Wise JP Sr. The cytotoxicity and genotoxicity of soluble and particulate cobalt in human lung fibroblast cells. Toxicol Appl Pharmacol. 2014;278(3):259-65.

27. Parsonage G, Filer AD, Haworth $O$, Nash GB, Rainger GE, Salmon M, et al. A stromal address code defined by fibroblasts. Trends Immunol. 2005;26(3): 150-6.

28. Stroka KM, Aranda-Espinoza $\mathrm{H}$. Endothelial cell substrate stiffness influences neutrophil transmigration via myosin light chain kinase-dependent cell contraction. Blood. 2011;118(6):1632-40.

29. Blakney AK, Swartzlander MD, Bryant SJ. The effects of substrate stiffness on the in vitro activation of macrophages and in vivo host response to poly (ethylene glycol)-based hydrogels. J Biomed Mater Res A. 2012;100(6):1375.

30. Belperio JA, Keane MP, Burdick MD, Lynch JP, Xue YY, Berlin A, et al. Critical role for the chemokine MCP-1/CCR2 in the pathogenesis of bronchiolitis obliterans syndrome. J Clin Invest. 2001;108(4):547-56.

31. Ferreira AM, Takagawa S, Fresco R, Zhu X, Varga J, DiPietro LA. Diminished induction of skin fibrosis in mice with MCP-1 deficiency. J Investig Dermatol. 2006;126(8):1900-8.

32. Dobaczewski M, Frangogiannis NG. Chemokines and cardiac fibrosis. Frontiers in bioscience (Scholar edition). 2009:1:391.

33. Fillingham YA, Della Valle CJ, Bohl DD, Kelly MP, Hall DJ, Pourzal R, et al. Serum metal levels for diagnosis of adverse local tissue reactions secondary to corrosion in metal-on-polyethylene Total hip Arthroplasty. J Arthroplast. 2017;32(9S):S272-S7

34. Kao C, Scalettar R, Bunning RD. Two cases of metallosis from metal-onpolyethylene total hips: an emerging problem. PM \& R: the journal of injury, function, and rehabilitation. 2015;7(4):447-50.

35. Hjorth MH, Mechlenburg I, Soballe K, Roemer L, Jakobsen SS, Stilling M. Higher prevalence of mixed or solid Pseudotumors in metal-onpolyethylene Total hip Arthroplasty compared with metal-on-metal Total hip Arthroplasty and resurfacing hip Arthroplasty. J Arthroplast. 2018;33(7): 2279-86.

36. Lawrence T, Gilroy DW. Chronic inflammation: a failure of resolution? Int J Exp Pathol. 2007:88(2):85-94

37. Jordana M, Sarnstrand B, Sime P, Ramis I. Immune-inflammatory functions of fibroblasts. Eur Respir J. 1994:7(12):2212-22.

\section{Publisher's Note}

Springer Nature remains neutral with regard to jurisdictional claims in published maps and institutional affiliations. 